\title{
Attribution of Foodborne Pathogens Using Structured Expert Elicitation
}

\author{
Arie H. Havelaar, ${ }^{1,2}$ Ángela Vargas Galindo, ${ }^{3, \star}$ Dorotha Kurowicka, ${ }^{3}$ and Roger M. Cooke ${ }^{3,4}$
}

\begin{abstract}
Objectives: To estimate the fraction of human cases of enterically transmitted illness by five major pathways (food, environment, direct animal contact, human-human transmission, and travel) and by 11 groups within the food pathway.

Methods: Food safety experts were asked to provide their estimates of the most likely range for each of the parameters. Joint probability distributions were created by probabilistic inversion (PI).

Results: Sixteen experts participated in the study. PI resulted in good fits for most pathogens. Qualitatively, expert estimates were similar to earlier published studies but the estimated fraction of foodborne transmission was lower for most pathogens. Biologically less plausible pathways were given some weight by the experts. Uncertainties were smallest for pathogens with dominant transmission routes.

Conclusions: Structured expert studies are a feasible method for source attribution, but methods need further development.

Applications: These estimates can be combined with data on incidence, disease burden and costs to provide specific estimates of the public health impact of foodborne illness, and to identify the food groups that have the highest impact.
\end{abstract}

\section{Introduction}

$\mathbf{P}$ RIORITY SETTING OF PATHOGENS that can (also) be transmitted by food is a complex process that includes evaluation of several indicators such as disease burden and cost of illness, which are typically available at an aggregate level. That is, the total burden and costs for a pathogen are calculated, irrespective of transmission route, see for example Havelaar et al. (2000, 2004, 2007) and Vijgen et al. (2007). For food safety policy, it is important to know which fraction of the total burden and costs are attributable to foods, and which foods are contributing to that fraction. Attribution of illness to different sources is a difficult process that can be based on different approaches that do not necessarily give the same answers, see for example Batz et al. (2005). These include outbreaks, analytical epidemiology (i.e., casecontrol studies), microbial subtyping, risk assessment, and intervention studies. No single approach may be expected to produce definitive data. Currently, the best possible approach is to combine data from these different approaches.

\footnotetext{
${ }^{1}$ Laboratory for Zoonoses and Environmental Microbiology, Centre for Infectious Disease Control Netherlands, National Institute for Public Health and the Environment, Bilthoven, The Netherlands.

${ }^{2}$ Division of Veterinary Public Health, Institute for Risk Assessment Sciences, Faculty of Veterinary Medicine, Utrecht University, Utrecht, The Netherlands.

${ }^{3}$ Applications of Decision Theory, Department of Applied Mathematics and Risk Analysis, Delft University of Technology, Delft, the Netherlands.

${ }^{4}$ Chauncey Starr Chair for Risk Analysis, Resources for the Future, Washington, DC

${ }^{*}$ Current address: PricewaterhouseCoopers Advisory, Amsterdam, The Netherlands.
} 
This necessitates subjective interpretation by a group of experts. The major challenge is to make this process transparent and acceptable to a broad group of stakeholders. We present a structured expert study to estimate the fraction of human cases of enterically transmitted illness by five major pathways (food, environment, direct animal contact, human-human transmission, and travel) and by 11 groups within the food pathway. As we did not consider precise estimates to be possible, uncertainty was quantified. A description of general principles of food attribution is followed by a description of the expert elicitation method. Data analysis by probabilistic inversion is described in general terms, as well as in detail as applied in this study. The results of the study are presented and discussed in the context of other published studies on attribution of foodborne pathogens.

\section{Methods}

\section{Principles of food attribution}

A two-step process was followed, firstly assessing the proportion of all cases that is foodborne and secondly assigning the proportion of foodborne cases to specific food groups. The total foodborne transmission was compared to three other major pathways as indicated in Fig. 1. The analysis took into account that exposure may take place in the country of residence but also during foreign travel. Such cases are typically considered as a separate category, because national hygiene legislation does not impact exposures abroad. Hence, the primary differentiation of exposure pathways included a separate category of travel-associated cases. A detailed description of these five pathways is given in Table 1.

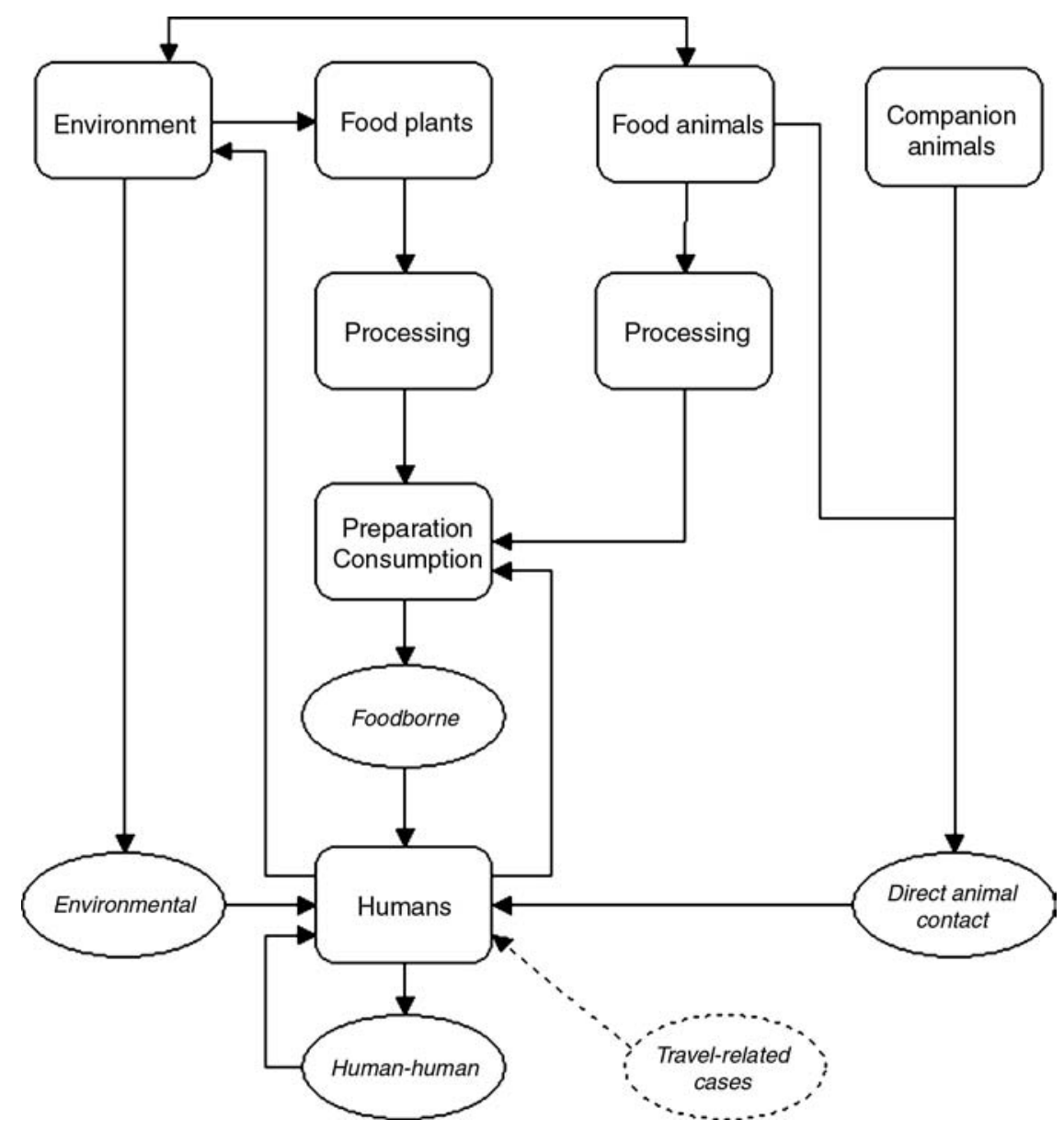

FIG. 1. Major transmission pathways for enteric pathogens. 
Table 1. Major Transmission Pathways and Food Groups for Attribution

\begin{tabular}{|c|c|}
\hline \multicolumn{2}{|l|}{ Major transmission pathways } \\
\hline Foodborne & $\begin{array}{l}\text { Transmission through food that is contaminated when it enters the } \\
\text { kitchen or during preparation (e.g., by food handlers) }\end{array}$ \\
\hline Environmental & $\begin{array}{l}\text { Transmission through contaminated water (drinking water, recreational } \\
\text { water), soil, air or other environmental media }\left(\text { fomites }^{\mathrm{a}}\right)\end{array}$ \\
\hline Human-human & Transmission from person to person by the fecal-oral route \\
\hline Direct animal & $\begin{array}{l}\text { Transmission by direct contact with live animals including pets, } \\
\text { farm animals, petting zoos, etc. }\end{array}$ \\
\hline Travel-associated & $\begin{array}{l}\text { Cases when exposure takes place by any of the above pathways } \\
\text { during foreign travel }\end{array}$ \\
\hline \multicolumn{2}{|l|}{ Food groups ${ }^{\mathrm{b}}$} \\
\hline Beef and lamb & $\begin{array}{l}\text { Beef, veal, lamb, and mutton. Includes processed and nonprocessed beef } \\
\text { products (sausages, filet américain [steak tartare], hamburgers, etc.) }\end{array}$ \\
\hline Pork & $\begin{array}{l}\text { Includes processed and nonprocessed pork products } \\
\text { (sausages, luncheon meats, etc.) }\end{array}$ \\
\hline $\begin{array}{l}\text { Chicken and other } \\
\text { poultry }\end{array}$ & $\begin{array}{l}\text { Includes duck, goose, ostrich, and turkey. Includes processed and } \\
\text { nonprocessed poultry products (chicken wings, marinated chicken, confits, etc.) }\end{array}$ \\
\hline Eggs & Including egg products \\
\hline Dairy products & Milk, cheese, butter, cream, etc. \\
\hline Fish and shellfish & $\begin{array}{l}\text { Includes all finfish, shellfish (mussels, oysters, etc.) and crustaceans } \\
\text { (lobster, shrimp, etc.) }\end{array}$ \\
\hline Fruit and vegetables & Includes (mixtures of) vegetables that are consumed raw or cooked \\
\hline Beverages & Includes all nonalcoholic and alcoholic beverages, except milk \\
\hline $\begin{array}{l}\text { Bread, grains, pastas, } \\
\text { and bakery products }\end{array}$ & Includes pastries \\
\hline $\begin{array}{l}\text { Other foods including } \\
\text { composite foods }\end{array}$ & $\begin{array}{l}\text { Includes all categories not listed above (e.g., nuts, oils, confectionery, spices) } \\
\text { and all foods that are sold to the consumer as a composite of two or more of } \\
\text { the above categories (e.g., pizzas, lasagna, nasi-goreng [fried rice], sandwiches) }\end{array}$ \\
\hline Infected humans or animals & Includes food handlers, vermin, pets, etc. \\
\hline
\end{tabular}

a Inanimate objects or substances capable of absorbing, retaining, and transporting contagious or infectious organisms (e.g., doorknobs and toys).

${ }^{\mathrm{b}}$ Contamination is assigned to the food category as it enters the kitchen. Cross-contamination to other products or the environment does not change this assignment (e.g., if chicken contaminates a salad, contamination is still assigned to chicken). Food contaminated by infected humans or animals during preparation is a separate category.

A more detailed examination of Fig. 1 shows that there are usually different transmission routes from a single reservoir. For example, if the reservoir of a pathogen is in food animals, transmission may be foodborne, environmental, or by direct contact. In this study, the point of exposure approach was used instead of the point of reservoir approach (Pires et al., submitted for publication).

Within the foodborne category, many subdivisions are possible. The Food Safety Research Consortium (http://www.thefsrc.org/) is developing the Foodborne Illness Risk Ranking Model (Batz et al., 2004). There are 14 major food categories and numerous subcategories for attribution in this model. Adak et al. (2005) have presented estimates of foodborne illness in England and Wales. These authors used a scheme with 10 major food groups and numerous food types. There are similarities as well as differences between these two schemes. At the level of major food categories or groups, the U.S. scheme is somewhat more disaggregated than the U.K. scheme. For example, the red meat category in the United Kingdom is split up into game, beef, and pork in the United States. There are also differences where a group of commodities is a major group in one scheme and a subgroup in the other. For example, rice and other dairy products are subgroups in the U.S. scheme and major groups in the U.K. scheme. Van Duynhoven et al. (2005) give annual reports on outbreaks of enteric illness in the Netherlands. The classification scheme used generally corresponds with but is more aggregated than the U.S. and U.K. schemes. For example, the Dutch scheme involves a category poultry and eggs that is disaggregated in the other schemes. Preferably, a classification scheme should not contain overlapping categories and if such categories are included, clear guidelines on classification must be given. Given the paucity of 
and sources of bias in the available data, it does not seem appropriate to use very detailed food groups. Based on these considerations, a simple classification scheme comprising 11 food groups was used in this study (see Table 1).

\section{The elicitation}

The experts invited to participate in this elicitation study included members from both industry and research (academia and institutes) with different scientific backgrounds including microbiology, epidemiology, and food science. Some experts had a broad perspective on food safety issues whereas other experts had studied particular foods or pathogens for several years, thus having a deeper but narrower knowledge.

The study included 17 pathogens that are currently being evaluated in a Dutch study on priority setting of foodborne pathogens (Kemmeren et al., 2006; Vijgen et al., 2007). Experts were presented with a detailed, written summary of the principles of attribution as applied to this study and the relevant (national and international) data, but were asked to evaluate possible biases in the data and the applicability to the Dutch situation themselves. Uncertainty was included by asking the experts to give an interval that contained, according to their assessment, the probability of transmission through a particular pathway or ingestion of a food group with $90 \%$ certainty. Appendix 1 gives an example of the questions included in the elicitation. Asking experts for intervals not only allows them to express their uncertainty but also reduces respondents' fatigue and allows them to answer questionnaires for more pathogens. The elicitation was done entirely by e-mail. After sending the invitation, experts were asked to indicate which combinations of pathogens and pathways and food groups they felt competent to complete. They were then sent a spreadsheet containing a separate form for each combination, which they completed electronically and returned by e-mail. Originally, 37 experts were invited and 28 agreed to participate. After the questionnaires were sent, two experts declined to participate, while 10 never sent their assessments. As a result, 16 experts participated in the elicitation. The number of experts participating per pathogen varied between 2 and 12.

\section{Data analysis}

For each pathogen and each pathway that pathogen might take, each expert gave an interval for which he or she is $90 \%$ certain contains the true probability that the given pathogen takes a certain pathway. Of course the probabilities of all pathways must sum to one, but the experts were not asked for their joint probability over all pathways. The joint probability complying with the experts' assessments for individual pathways was constructed in the manner described below.

Illustrative data from three experts are shown in Fig. 2, for pathway $X$ and for pathogen $Y$. The intervals are converted to density functions, also shown in Fig. 2. These densities are the minimally informative densities complying with each expert's $90 \%$ interval. Combining these densities with equal weight results in the bold black line in Fig. 3. Suppose we now have these equal weight decision maker (EWDM) combinations for each of the five pathways that pathogen $Y$ can take. Each individual density represents the combined uncertainty over the probability that this pathway is chosen.

The next step is to convert these distributions over each pathway's probability into a distribution over the set of probability vectors of

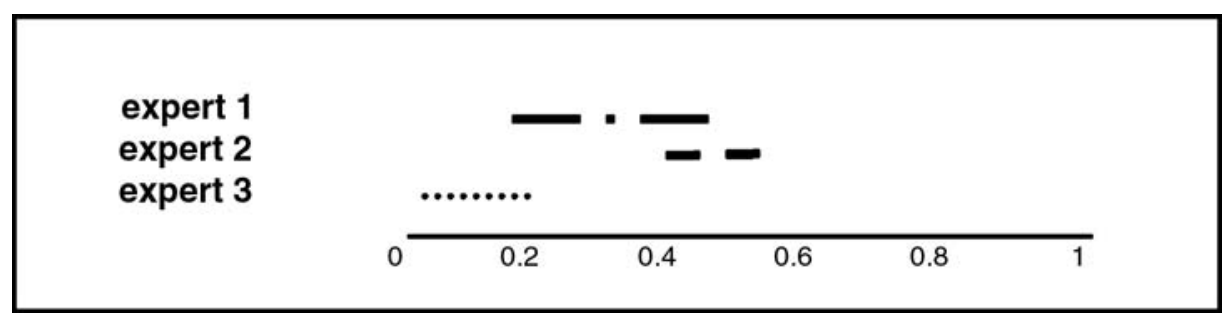

FIG. 2. Three experts' $90 \%$ intervals for the probability that pathogen $\mathrm{X}$ takes pathway $\mathrm{Y}$. 


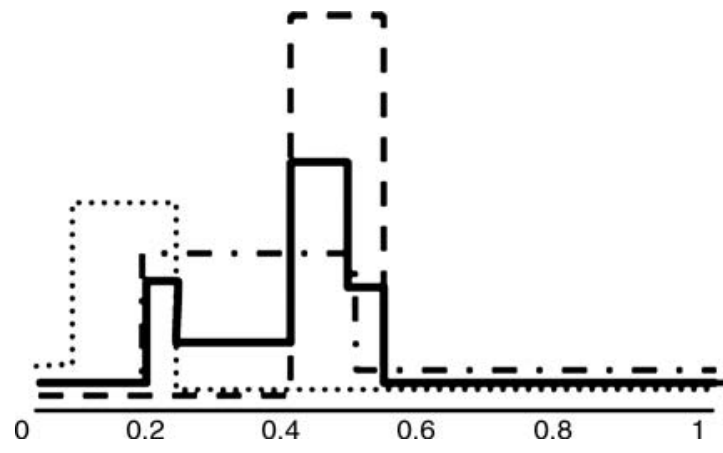

FIG. 3. Equal weight combination (solid) of three experts' density functions (dashed) that pathogen $X$ takes pathway Y.

length 5 . There may be substantial uncertainty surrounding each pathway, but we know that the sum of the probabilities over all pathways must equal 1, since only one pathway is chosen in each instance of the disease.

The method for accomplishing this is called probabilistic inversion. Further details are given in Appendix 2.

\section{Results}

The number of experts who completed a questionnaire varied between 12 and 2 per pathogen, and was somewhat higher for food groups than for major pathways. Even though the present elicitation lacks seed variables that would enable the analyst to filter some experts (Cooke, 1995), there was enough evidence to consider filtering one of the experts as these results were negatively affecting the Decision Maker (DM) distribution but also the interpretation of the questions in the elicitation was not considered correct. After filtering this expert, the results in general were satisfactory. To illustrate the method, Table 2 shows the probabilistic inversion results for Campylobacter spp. The 5\% and $95 \%$ columns under DM are the percentiles of each pathway that we would like to recover with probabilistic inversion. The 5\% and $95 \%$ columns under "Resampling" show the results of the probabilistic inversion. The "Estimated fraction" is the mean of the resampled probabilities, for each pathway. Note that these fractions sum to one, as required. The results in Table 2 are quite good.

The results of fitting the $5 \%$ and $95 \%$ quantiles for the 17 pathogens are summarized in Tables 3 and 4 . The fit was good enough for the majority
Table 2. Probabilistic Inversion Results for Major PATHWAYS OF CAMPYLOBACTER SPP.

\begin{tabular}{|c|c|c|c|c|c|}
\hline \multirow[t]{2}{*}{ Pathways } & \multirow[t]{2}{*}{$\begin{array}{l}\text { Estimated } \\
\text { fraction }\end{array}$} & \multicolumn{2}{|c|}{$D M$} & \multicolumn{2}{|c|}{ Resampling } \\
\hline & & $5 \%$ & $95 \%$ & $5 \%$ & $95 \%$ \\
\hline Food & 0.4210 & 0.2270 & 0.852 & 0.158 & 0.837 \\
\hline Environment & 0.2060 & 0.0003 & 0.7310 & 0.0003 & 0.7310 \\
\hline Human & 0.0628 & 0.0001 & 0.1230 & 0.004 & 0.1230 \\
\hline Animal & 0.1910 & 0.0022 & 0.5980 & 0.0022 & 0.05980 \\
\hline \multirow[t]{2}{*}{ Travel } & 0.1200 & 0.0020 & 0.2840 & 0.0022 & 0.2880 \\
\hline & \multicolumn{2}{|c|}{ No. of experts } & 12 & Method & IPF \\
\hline
\end{tabular}

DM, Decision Maker; IPF, iterative proportional fitting. See text for explanation.

of the pathogens, being slightly better for the pathways compared to the food groups. This is due to the number of elements. It is simpler for both the assessment and the analysis step to manage a smaller number of items. For the experts it is easier to give estimates for a smaller number of items, because it is easier to compare fewer options and it is easier to be aware of probabilities that are of bigger magnitude. Naturally, as the number of items increases the comparisons and the assessments are harder because the analysis demands from the expert a more accurate knowledge and a larger capability of taking all the options into account.

Evaluation of the 5th and 95th percentiles in Tables 3 and 4 shows that for most estimates, there is considerable uncertainty. Experts' individual estimates did not agree in many cases which results in wide uncertainty intervals in the absence of seed variables. The broad intervals for parameter estimates may be considered to reflect current uncertainty about the proportion of pathogens that is transmitted by food or specific food groups. Note that for pathogens that are typically considered to be almost exclusively foodborne (e.g., Clostridium perfringens and Staphylococcus aureus), the $90 \%$ intervals for major pathways are much smaller than for typical multi-source pathogens such as Campylobacter and STEC O157. For food groups, the uncertainty is smaller for pathogens with a single reservoir, e.g., viruses (humans, pigs) in comparison to $S$. aureus.

\section{Discussion}

Because of a lack of definitive data, there is no way to formally validate the results. One option 
Table 3. Transmission of 17 Enteropathogens by Major Pathways

\begin{tabular}{|c|c|c|c|c|c|c|}
\hline \multirow[b]{2}{*}{ Pathogen } & \multirow[b]{2}{*}{ Experts } & \multicolumn{5}{|c|}{ Fraction $(\%)$ transmitted by pathway ${ }^{\mathrm{a}}$} \\
\hline & & Food & Environment & Human & Animal & Travel \\
\hline Campylobacter spp. & 12 & $42(16-84)$ & $21(0-73)$ & $6(0-12)$ & $19(0-60)$ & $12(0-29)$ \\
\hline Shiga toxin-producing E. coli & & & & & & \\
\hline O157 & $\begin{array}{l}3 \\
3\end{array}$ & $40(15-83)$ & $17(0-47)$ & $10(0-23)$ & $21(0-76)$ & $\begin{array}{r}12(0-27) \\
6(0-10)\end{array}$ \\
\hline Non-O157 & 3 & $42(21-78)$ & $14(0-29)$ & $10(0-20)$ & $28(11-48)$ & $6(0-10)$ \\
\hline Listeria monocytogenes & 7 & 69 (47-98) & $7(0-18)$ & $5(0-13)$ & $5(0-13)$ & $13(0-40)$ \\
\hline Mycobacterium avium & 4 & $42(0-79)$ & $19(0-58)$ & $18(0-57)$ & $9(0-27)$ & $12(0-39)$ \\
\hline Salmonella spp. & 8 & $55(32-88)$ & $13(0-29)$ & $9(0-19)$ & $9(0-19)$ & $14(3-26)$ \\
\hline Bacillus cereus toxin & 4 & $90(68-100)$ & $1(0-4)$ & $1(0-4)$ & $1(0-4)$ & $7(0-91)$ \\
\hline Clostridium perfringens toxin & 4 & $91(72-100)$ & $2(0-5)$ & $2(0-5)$ & $2(0-5)$ & $3(0-9)$ \\
\hline Staphylococcus aureus toxin & 4 & $87(73-100)$ & $4(0-9)$ & $3(0-8)$ & $2(0-5)$ & $4(0-10)$ \\
\hline Enterovirus & 2 & $6(0-16)$ & $25(0-60)$ & $60(30-92)$ & $2(0-2)$ & $7(0-15)$ \\
\hline Hepatitis A virus & 2 & $11(0-20)$ & $11(0-19)$ & $18(0-42)$ & $0(0-0)$ & $60(7-80)$ \\
\hline Hepatitis E virus & 2 & $14(0-38)$ & $25(0-75)$ & $8(0-20)$ & $11(0-29)$ & $43(0-68)$ \\
\hline Norovirus & 5 & $17(16-47)$ & $14(0-43)$ & $55(42-88)$ & $5(0-10)$ & $9(0-20)$ \\
\hline Rotavirus & 3 & $13(13-28)$ & $17(0-46)$ & $58(43-90)$ & $3(0-5)$ & $9(0-19)$ \\
\hline Cryptosporidium parvum & 2 & $12(0-20)$ & $28(10-39)$ & $27(10-38)$ & $13(5-19)$ & $20(4-29)$ \\
\hline Giardia lamblia & 3 & $13(0-24)$ & $24(10-37)$ & $35(10-56)$ & $11(0-20)$ & $18(5-29)$ \\
\hline Toxoplasma gondii & 3 & $56(26-88)$ & $36(6-66)$ & $1(0-1)$ & $3(0-3)$ & $5(0-9)$ \\
\hline
\end{tabular}

${ }^{\mathrm{a}}$ Mean (5th and 95th percentile) after resampling. Bold type indicates poor fit with Decision Maker.

is to compare the results with those from other (usually less comprehensive) studies. Complete studies that break down transmission pathways in the above-mentioned categories are not available in the literature. Some authors have provided estimates of the fraction of foodborne illness. Table 5 gives an overview of published (international) data for the 17 pathogens that are included in this study. At first glance the results of the present study appear logical. The pathogens can be grouped in several categories with respect to their major pathways. Toxins (Bacillus cereus [Bc], Clostridium perfringens [Cp], Staphylococcus aureus [Sa]) are predominantly considered foodborne whereas other bacterial pathogens (Campylobacter spp. [Ca]; STEC O157 and non-O157 [STEC]; Listeria monocytogenes [Lm]; Mycobacterium avium [Ma]; Salmonella spp. [Sa]) and the protozoan parasite Toxoplasma gondii (Tg) are considered to be transmitted by multiple pathways with food as the most important one. The other protozoan parasites (Cryptosporidium parvum [Cp] and Giardia lamblia [Gl]) are considered to be predominantly transmitted between humans and through the environment with a small fraction by food. A similar fraction of viruses is considered to be foodborne with mainly transmission between humans (enterovirus [Ev], norovirus $[\mathrm{Nv}]$, rotavirus $[\mathrm{Rv}]$ ) or during travel (hepatitis-A virus [Hav] and hepatitis-E virus [Hev]). From a qualitative point of view, these estimates are consistent with the general epidemiological data on these pathogens. While results may be expected to vary internationally due to differences in food production and consumption, it is worth noting that for most pathogens the fraction transmitted by food is considerably lower than other international estimates (cf. Table 5). The data are closer to an earlier Dutch report, but also in comparison with these data the proportion of cases of salmonellosis in the present study is low ( $55 \%$ vs. $>90 \%$ ). Several pathogens are attributed in low percentages $(<5 \%)$ to pathways that are biologically implausible. This is the case for Bc, Cp, Sa, and Lm by the environment, humans and animals as growth of these bacteria is usually required to produce illness. Direct transmission of $\mathrm{Tg}$ by humans or animals is biologically impossible as humans do not excrete infectious oocysts and those excreted by felines need to maturate in the environment for several days before becoming infectious. There is no evidence that $\mathrm{Ev}, \mathrm{Nv}$, and $\mathrm{Rv}$ are zoonotic, hence transmission by animals is unlikely.

Within the food pathway, results are also generally consistent with the overall epidemiology of the pathogens. Beef and lamb are considered the main food involved in transmission of STEC and Cp, poultry of $\mathrm{Ca}$, and pork of 


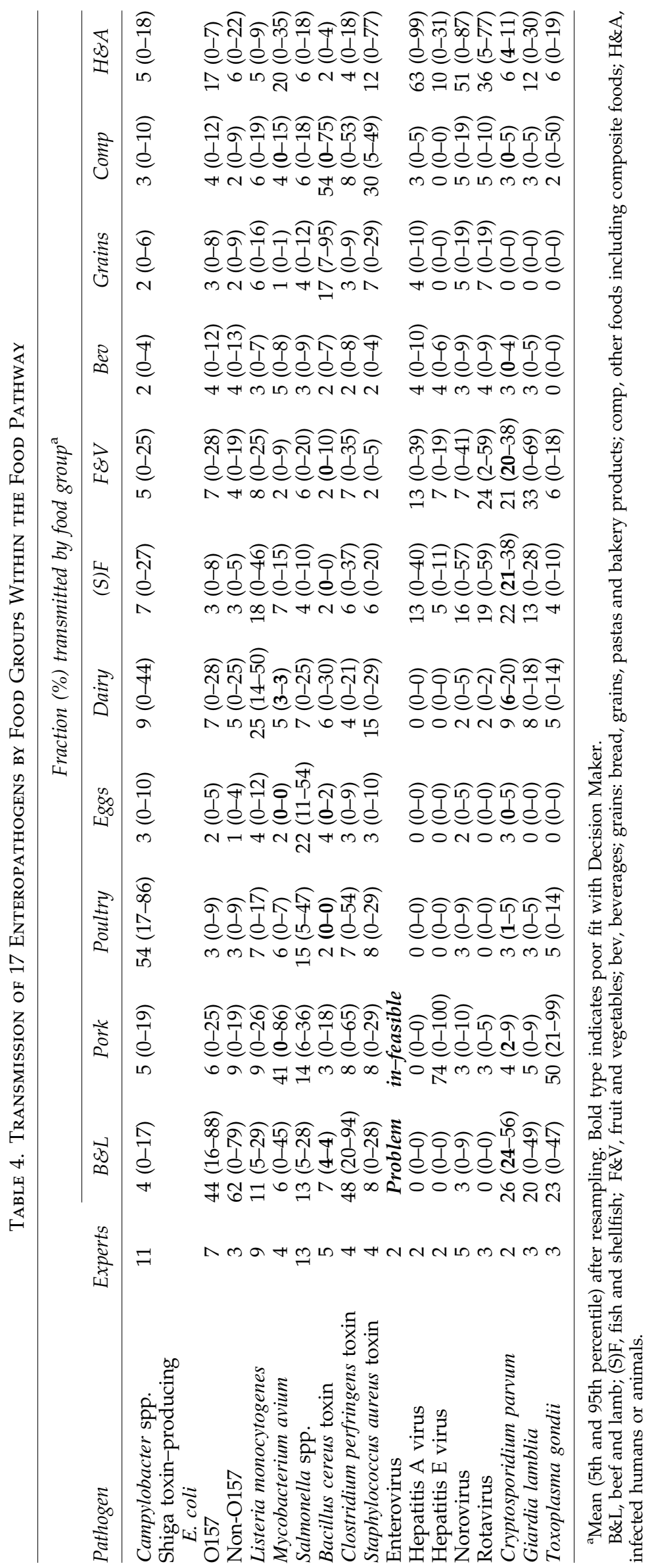


Table 5. Proportion of Illness Cases That Are Attributed to Food

\begin{tabular}{|c|c|c|c|c|c|c|}
\hline & \multicolumn{6}{|c|}{ Reference $^{\mathrm{a}}$} \\
\hline & Mead & Adak & Duyn & Anon & Hall & This study \\
\hline Country & USA & UK & NL & France & Australia & NL \\
\hline Period & 1990s & 1992-2000 & $1990 \mathrm{~s}$ & 1990s & 2000 & 2006 \\
\hline Data sources ${ }^{b}$ & $\mathrm{E} / \mathrm{O} / \mathrm{R}$ & $\mathrm{O}$ & $\mathrm{E} / \mathrm{CC}$ & $\mathrm{E}$ & $\mathrm{E}$ & $\mathrm{E}$ \\
\hline Travel-related cases & Included & Excluded & Included & Included & Excluded & Included \\
\hline Campylobacter spp. & $80 \%$ & $80 \%$ & $30-80 \%$ & $80 \%$ & $75 \%$ & $42 \%$ \\
\hline \multicolumn{7}{|l|}{ Shiga toxin-producing $E$. coli } \\
\hline O157 & $85 \%$ & $63 \%$ & $50-90 \%$ & $50 \%$ & $65 \%$ & $40 \%$ \\
\hline Non-O157 & $85 \%$ & $\mathrm{NA}^{\mathrm{c}}$ & NA & NA & $65 \%$ & $42 \%$ \\
\hline Listeria monocytogenes & $99 \%$ & $99 \%$ & & $99 \%$ & NA & $69 \%$ \\
\hline Mycobacterium avium & NA & NA & NA & NA & NA & $42 \%$ \\
\hline Salmonella spp. & $95 \%$ & $92 \%$ & $>90 \%$ & $95 \%$ & $87 \%$ & $55 \%$ \\
\hline Bacillus cereus toxin & $100 \%$ & $100 \%$ & $100 \%$ & $100 \%$ & $100 \%$ & $90 \%$ \\
\hline Clostridium perfringens toxin & $100 \%$ & $94 \%$ & $100 \%$ & $100 \%$ & $100 \%$ & $91 \%$ \\
\hline Staphylococcus aureus toxin & $100 \%$ & $96 \%$ & $100 \%$ & $100 \%$ & $100 \%$ & $87 \%$ \\
\hline Enterovirus & NA & NA & NA & NA & NA & $6 \%$ \\
\hline Hepatitis A virus & $50 \%$ & NA & NA & $5 \%$ & NA & $11 \%$ \\
\hline Hepatitis E virus & NA & NA & NA & NA & NA & $14 \%$ \\
\hline Norovirus & $40 \%$ & $11 \%$ & $10-20 \%$ & $14 \%$ & $25 \%$ & $17 \%$ \\
\hline Rotavirus & $1 \%$ & $3 \%$ & $0-10 \%$ & NA & $2 \%$ & $13 \%$ \\
\hline Cryptosporidium parvum & $10 \%$ & $6 \%$ & ?? & NA & $10 \%$ & $12 \%$ \\
\hline Giardia lamblia & $10 \%$ & $10 \%$ & $<30 \%$ & NA & $5 \%$ & $13 \%$ \\
\hline Toxoplasma gondii & $50 \%$ & NA & NA & $50 \%$ & NA & $56 \%$ \\
\hline
\end{tabular}

${ }^{a}$ Mead, Mead et al. (1999); Adak, Adak et al. (2002); Duyn, Van Duynhoven et al. (2002); Anon, Anonymous (2004); Hall, Hall et al. (2005).

${ }^{\mathrm{b}} \mathrm{E}$, expert estimates; $\mathrm{O}$, outbreaks; R, reported cases; CC, case-control studies.

'NA, not applicable.

$\mathrm{Ma}, \mathrm{Hev}$, and $\mathrm{Tg}$ (the latter with a substantial contribution by beef and lamb). Sa is the only pathogen that is attributed to eggs in a substantial amount, with all meat groups also considered important. The pathogens for which humans are the only reservoirs (Hav, Nv, Rv) are mainly attributed to infections by humans and animals with (shell)fish and fruits and vegetables as secondary food groups. These latter groups are also considered of importance for the $\mathrm{Cp}$ and $\mathrm{Gl}$, which are also attributed to beef and lamb.

Quantitatively, it is noteworthy that for the specific human pathogens Hav and Rv, the foods of animal origin receive a score of $0 \%$; while Hev is only attributed to pork, consistent with its detection in slaughter pigs. For Nv, the experts are less consistent in their dismissal of zoonotic transmission. Bc and Sa are mainly attributed to composite foods with secondary routes being bread, grains, etc. for $\mathrm{Bc}$ and dairy for Sa. Again, this is consistent with the general epidemiology.

To evaluate in how far the relatively high scores attributed to less plausible routes were related to the chosen methods, additional data analysis was carried out. First, the probabilistic inversion was repeated with addition of a fit to the 50th percentile (arbitrarily set as the midpoint between the 5th and 95th percentiles). Secondly, some biologically implausible results were defined as seed variables (Cooke, 1995) and the experts were weighed according to their score on these seed variables. For both methods, the results were hardly different from the baseline model and it is concluded that the scores were not a result of the data analysis but did represent the experts' beliefs.

As for pathways, it is remarkable, that biologically less plausible routes do score a small percentage of transmission, e.g., Ca in bread, grains, etc. (Ca survives very poorly on dry products) and several pathogens in eggs (only two Salmonella serovars are able to invade the oviduct of laying hens).

Hoffmann et al. (2007) recently published an expert survey on attribution in the United States. The study was limited to the food pathway. Experts were asked for their best estimates and $90 \%$ confidence bounds. Weighted means 
were reported and combined with incidence estimates of foodborne illness as published by Mead et al. (1999). Uncertainty estimates and their implications are discussed in a separate paper (Hoffmann et al., in press). Results are difficult to compare with the present study because of different food groupings and different definitions. For example, in the U.S. study, a salad that was cross-contaminated during preparation was assigned to the produce group in the U.S. study, but was assigned to the contaminated food entering the kitchen (say chicken) in our study. Such details in definitions should be carefully considered by decision makers. Results should not be expected to be similar as the epidemiology of foodborne illness differs between the United States and the Netherlands. For example, experts assign a far greater role to produce in the United States, which is in line with the far greater number of reported outbreaks. On the other hand, eggs are considered to be the dominant source of salmonellosis in the Netherlands, in contrast to poultry in the United States. Similar results are obtained, for example, for the importance of poultry in the transmission of campylobacteriosis and of beef for STEC O157.

The response rate in our study was 43\% (16/ 37). The background of the invited experts was in microbiology (25), epidemiology (5), and food science (7); the respondents were 11 microbiologists, 4 epidemiologists, and 1 food scientist. Thus, while among the invitations, microbiologists were overrepresented, this was less so among the respondents, but food scientists were underrepresented. The low response may have been due to a high burden to complete all data sheets. We have no information on a possible influence on our results by the nonresponse.

An expert study may not be expected to provide an unbiased estimate of the relative importance of different transmission routes. Rather, it should be regarded as a structured way to obtain a consensus opinion, based on evaluation of all available data. For policymaking purposes, this consensus opinion may be highly valuable. Future developments should aim at developing more data-driven approaches, combining information from different sources in a single analytical framework.
To provide more comparable data, international harmonization of definitions, groupings, protocols, and data analysis methods should be aimed for.

\section{Acknowledgments}

The authors are grateful to the experts for participation in this study. Yvonne van Duynhoven, Wilfrid van Pelt, and Eric Evers (RIVM) provided feedback on the study design. This study was funded by the Dutch Ministry of Public Health, Welfare and Sports, the Hague, the Netherlands.

\section{References}

Adak GK, Long SM, and O'Brien SJ. Trends in indigenous foodborne disease and deaths, England and Wales: 1992 to 2000. Gut 2002;51:832-841.

Adak GK, Meakins SM, Yip H, Lopman BA, and O'Brien SJ. Disease risks from foods, England and Wales, 19962000. Emerg. Infect. Dis. 2005;11:365-372.

Anonymous. 2004. Morbidité et mortalité dues aux maladies infectieuses d'origine alimentaire en France. Saint-Maurice, France: Institut National de Veille Sanitaire.

Batz MB, Doyle MP, Morris G, Painter J, Singh R, Tauxe RV, Taylor MR, and Lo Fo Wong DMA. Attributing illness to food. Emerg. Infect. Dis. 2005;11:993-999.

Batz MB, Hoffmann SA, Krupnick AJ, Morris JG, and Taylor MR. Identifying the most significant microbiological foodborne hazards to public health: a new risk ranking model. Food Safety Research Consortium Discussion paper number 1. Available at http:// www.thefsrc.org/Discussion\%20Papers/FRSC-DP01. pdf. Washington, D.C.: Resources for the Future, 2004.

Cooke RM. Experts in Uncertainty. Oxford: Oxford University Press, 1995.

Cooke RM, Nauta M, Havelaar AH, and Van der FelsKlerx I. Probabilistic inversion for chicken processing lines. Reliability Engineering and System Safety 2006; 91:1364-1372.

Hall G, Kirk MD, Becker N, Gregory JE, Unicomb L, Millard G, et al. Estimating foodborne gastroenteritis, Australia. Emerg. Infect. Dis. 2005;11:1257-1264.

Havelaar AH, De Wit MAS, Van Koningsveld R, and Van Kempen E. Health burden in the Netherlands due to infection with thermophilic Campylobacter spp. Epidemiol. Infect. 2000;125:505-522.

Havelaar AH, Kemmeren JM, and Kortbeek LM. Disease burden of congenital toxoplasmosis. Clin. Infect. Dis. 2007;44:1467-1474.

Havelaar AH, Van Duynhoven YTHP, Nauta MJ, Bouwknegt M, Heuvelink AE, De Wit GA, Nieuwenhuizen MGM, and Van de Kar NCAJ. Disease burden in the Netherlands due to infections with Shiga-toxin 
producing Escherichia coli O157. Epidemiol. Infect. 2004;132:467-484.

Hoffmann S, Fischbeck P, Krupnick A, and McWilliams M. Elicitation from large, heterogeneous expert panels: using multiple uncertainty measures to characterize information quality for decision analysis. Decision Analysis: in press.

Hoffmann S, Fischbeck P, Krupnick A, and McWilliams M. Using expert elicitation to link foodborne illnesses in the United States to foods. J. Food Prot. 2007;70:1220 1229.

Kemmeren JM, Mangen M-JJ, Van Duynhoven YTHP, and Havelaar AH. Priority setting of foodborne pathogensdisease burden and costs of selected enteric pathogens. Bilthoven, the Netherlands: National Institute for Public Health and the Environment, 2006.

Mead PS, Slutsker L, Dietz V, Mccaig LF, Bresee JS, Shapiro C, et al. Food-related illness and death in the United States. Emerg. Infect. Dis. 1999;5:607-625.

Van Duynhoven YTHP, De Boer IM, and Van den Broek MJM. Registratie van voedselinfecties en -vergiftigingen bij de Inspectie voor de Gezondheidszorg en Voedsel en Waren Autoriteit, 2004. Bilthoven, the Netherlands: Rijksinstituut voor Volksgezondheid en Milieu, 2005.
Van Duynhoven YTHP, De Wit MAS, Kortbeek LM, and Koopmans MPG. Voedselinfecties in Nederland. Ned. Tijdschr. Med. Microbiol. 2002;10:79-83.

Vijgen SMC, Mangen MJM, Kortbeek LM, Van Duynhoven YTHP, and Havelaar AH. Disease burden and related costs of cryptosporidiosis and giardiasis in the Netherlands. Bilthoven, the Netherlands: National Institute for Public Health and the Environment, 2007.

Address reprint requests to: Arie H. Havelaar, Ph.D., M.Sc.

Laboratory for Zoonoses and Environmental Microbiology

The Netherlands Centre for Infectious Disease

Control Netherlands

National Institute for Public Health and

the Environment

3720 BA Bilthoven

P.O.Box 1

The Netherlands

E-mail: arie.havelaar@rivm.nl

\section{APPENDIX 1}

Example Excel $^{\circledR}$ Sheet for Expert Elicitation

SALMONELLA (Pathways)

Expert's Information

Name

Email address

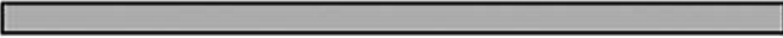

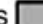

For each event, please specify an interval which contains the probability of occurrence with $90 \%$ certainty.

1. A case of illness due to Salmonella was transmitted by food ingestion.

2. A case of illness due to Salmonella was transmitted through the environment.

3. A case of illness due to Salmonella was transmitted by contact with a sick person. $0 \%$

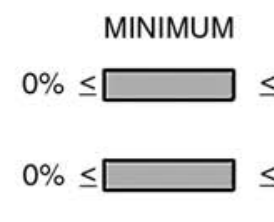

$\leq$

MAXIMUM

4. A case of illness due to Salmonella was transmitted by direct animal contact.

5. A case of illness due to Salmonella was transmitted abroad by any pathway.

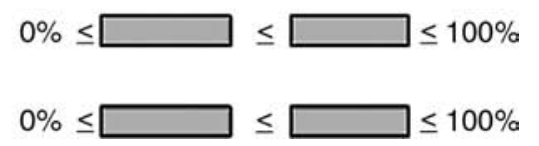




\section{APPENDIX 2}

We first describe a simple implementation of the probabilistic inversion method and then indicate how this simple method was adapted to deal with particular problems.

We first draw a large sample of probability 5 vectors, say 100,000 . This is simply a list of numbers $\left(p_{1}, \ldots, p_{5}\right)$, such that $p_{i} \geq 0$ and $\Sigma_{i} p_{i}=1$. There are many ways to do this, a convenient method is to sample independent gamma variables $g_{1}, \ldots, g_{5}$ with the same shape and scale parameters, and then define, on each sample

$$
p_{i}=g_{i} / \Sigma_{j} g_{j}
$$

If we now consider the marginal distribution of, for example, $p_{2}$ in this list of 100,000 vectors, this will not agree with the distribution in Fig. 2. The idea is now to re-weight this sample so that the marginal distributions of $p_{1}, \ldots p_{5}$ in the reweighted sample fit as closely as possible the distributions of the EWDM. The method for finding these weights is called iterative proportional fitting (IPF), and a description of this method, and some of its variations, may be found in Cooke et al. (2006). There is no reason why there should be a distribution over probability 5 vectors whose margins agree exactly with those from the EWDM. Indeed, the experts were asked only for marginal distributions of probabilities; asking the experts to insure that these margins were consistent with a joint distribution over probability 5 vectors would impose a daunting task. Moreover, the simplifications involved in constructing the densities in Fig. 2 might undo any such effort. Hence, it may happen that there is no distribution over probability 5 vector that exactly recovers the
EWDM's distributions. The probabilistic inversion in such cases is infeasible. Where infeasibility was an issue, the PARFUM variant of IPF was applied, as this algorithm generally has better behavior in case of infeasibility.

To do the probabilistic inversion with fully specified densities would require sample sizes beyond the capability of current platforms. To keep the sample sizes manageable, we approximated each density as a piece-wise uniform density whose 5th and 95th percentiles agreed with the equal weight decision maker's density. We compared the results to the results obtained by adding the (interpolated) 50\% value, and found little difference. Even with this simplification it is not always possible to find such a set of weights: that is, it may not be possible to recover all the marginal distributions in this way. When this happens we may either draw a larger sample, or sample the starting $p_{i}$ 's more intelligently. In particular, if we know that the mean of the distribution in Fig. 3 is 0.35, then we could choose the shape factors of our gamma distributions differentially (the scale factors must be the same). If the shape factor of $g_{i}$ is $a_{i}$, then we can arrange that $a_{3} / \Sigma_{j} a_{j}=0.35$. This will insure that the mean of $p_{i}$ in the starting distribution, before re-weighting, is 0.35 , and this will make an "infeasible" problem "less infeasible" without increasing the sample size. In cases of difficult fitting, such techniques were employed, but most of the fitting problems were feasible, or nearly feasible, without such special nursing.

There were some cases when there was absolute consensus among the group of experts. In these cases, where all experts agreed that the probability of contamination is equal to zero the respective random variable was deleted beforehand. 
